\title{
AVALIAÇÃO DE UM WETLAND CONSTRUIIDO HÍBRIDO NO TRATAMENTO DE ESGOTO SANITÁRIO
}

\author{
EVALUATION OF A WETLAND CONSTRUCTED \\ HYBRID IN SEWAGE TREATMENT SANITARY
}

\author{
Marina Victoretti Silva ${ }^{1}$; Rodrigo Freitas Bueno ${ }^{2}$ \\ ${ }^{1}$ Universidade Federal do ABC. Avenida dos Estados, 5001 - Bangú, Santo André - \\ SP, 09210-580. E-mail: marinavictoretti@ hotmail.com \\ ${ }^{2}$ Escola Politécnica da Universidade de São Paulo. Av. Prof. Luciano Gualberto, 380 \\ - Butantã, São Paulo - SP, 05508-010. E-mail: robueno@usp.br.
}

\begin{abstract}
RESUMO
No Brasil, há um déficit de acesso em relação aos serviços de esgotamento sanitário por grande parte da população, sendo esse fato um dos responsáveis pela contaminação de corpos hídricos. Isso se dá devido à falta de investimento em infraestrutura no país, uma vez que estações de tratamento de esgoto demandam custos elevados e grande demanda de área para implantação, além da instalação do sistema de coleta, que encaminha o esgoto sanitário ao tratamento. Sendo assim, é necessário então, que sejam desenvolvidas tecnologias acessíveis nesse setor, visando à disseminação deste serviço. Dentro deste contexto, os Wetlands Construídos podem ser considerados uma alternativa de tratamento descentralizado de esgotos, de baixo custo, esteticamente e ecologicamente viáveis. Este estudo, portanto, avaliou a aplicabilidade de um sistema composto por Wetland Construído Híbrido - WCH (fluxo vertical seguido de horizontal), em escala piloto, tratando esgoto sanitário. $\mathrm{O} \mathrm{WCH}$ apresentou eficiências satisfatórias em termos de remoção de matéria orgânica, reduzindo turbidez e sólidos de maneira eficaz, porém apresentou baixa eficiência em termos de remoção de nutrientes. As baixas temperaturas registradas nas seis primeiras semanas limitaram o crescimento das macrófitas, contudo, nas semanas seguintes houve desenvolvimento expressivo. Assim, concluiu-se que o $\mathrm{WCH}$ pode ser uma alternativa para o tratamento de esgotos sanitários, podendo ser considerado como um sistema técnica e economicamente viável para disseminação dos serviços de esgotamento sanitário, principalmente, visando pequenas comunidades.
\end{abstract}

Palavras-chave: Wetlands Construídos. Esgoto sanitário. Remoção de matéria orgânica. Remoção de nitrogênio.

\footnotetext{
ABSTRACT

In Brazil, there is a deficit of access in relation to sewage services for a large part of the population, and this fact is the one responsible for the contamination of watercourses. This is because to the lack of investment in infrastructure in the country, since sewage treatment plants require high costs and high area demand for deployment, besides the installation of the collection system, which directs the wastewater to treatment. Therefore, it is necessary that accessible technologies be developed in this sector, aiming to disseminate this service. Inside this context, Constructed Wetlands can be considered an alternative of decentralized wastewater treatment, inexpensive,
} 
aesthetically and ecologically viable. Thus, this study evaluated the applicability of a system composed by Hybrid Constructed Wetland (HCW) (vertical by horizontal follow) in a pilot scale treating domestic sewage. The WCH showed satisfactory efficiencies in terms of organic matter removal, reducing turbidity and solids effectively, but showed low efficiency in terms of nutrient removal. The low temperatures recorded in the first six weeks limited the growth of macrophytes; however, in the following weeks there was expressive development. Thus, it was concluded that the application of $\mathrm{WCH}$ for wastewater treatment is viable and can be considered a technically and economically alternative for disseminate the sanitation services, mainly aimed at small communities.

Keywords: Constructed Wetlands. Wastewater. Organic matter removal. Nitrogen removal.

\section{INTRODUÇÃO}

A qualidade da água é resultante de fenômenos naturais e da atuação do homem. De maneira geral, pode-se dizer que a qualidade de uma determinada água é função das condições naturais do uso e da ocupação do solo na bacia hidrográfica. No país há um grande déficit de investimentos na área de saneamento básico, principalmente, quanto às estações de tratamento de esgotos, que representam um dos grandes investimentos com alto custo de capital, além de custo de operação e manutenção. Esse cenário fica mais crítico quando se pensa nas comunidades isoladas (loteamentos ou núcleos habitacionais localizados normalmente em áreas periféricas de cidades, ou comunidades, litorâneas ou não) onde a implantação de grandes estações de tratamento de esgotos como sistemas de lodos ativados fica impraticável.

Dentro deste contexto, tem aumentado o interesse pela descentralização de processos de tratamento de esgotos por sistemas modulares com baixo custo, economicamente viáveis, estética e ecologicamente sustentáveis com capacidade de restaurar ambientes degradados e, ao mesmo tempo, serem integrados aos ciclos naturais como o ciclo da água (CRITES et al., 1998; MASSOUD et al., 2009; MORAES, 2012).

As condições climáticas no Brasil favorecem a aplicação de sistemas naturais, onde se destacam os sistemas naturais de tratamento de esgoto conhecido como Wetlands Construídos (WC), que podem ser aplicados de acordo com o contexto local e apresentam diversas vantagens como: auxiliar na gestão integrada dos recursos hídricos; reciclagem de nutrientes e a diminuição da entrada, por exemplo, de nitrogênio e fósforo nos corpos hídricos; auxilia no ciclo da água, promovendo a recarga de aquíferos; redução dos impactos das enchentes na drenagem urbana; melhoria do microclima local com a recuperação de habitats em áreas próximas à córregos, rios e represas (BRIX, 1994; COOPER, 1999; COOPER et al., 1999; DIERBERG et al., 2002; BRIX e ARIAS, 2005). Assim, este trabalho torna-se relevante em avaliar oportunidades de desenvolvimento e inovação nas áreas de interface entre recursos hídricos e saneamento ambiental.

\section{MATERIAL E MÉTODOS}

\subsection{Descrição geral do experimento}

O estudo foi realizado nas dependências do Centro Tecnológico de Hidráulica e Recursos Hídricos (CTH) localizado na Escola Politécnica da Universidade de São Paulo (EPUSP), São Paulo - Brasil. As atividades de laboratório foram realizadas no Laboratório de Saneamento Prof. Lucas Nogueira Garcez - EPUSP. O esgoto que alimentou o sistema foi proveniente do Conjunto Residencial (CRUSP) e do Restaurante Central da Universidade de São Paulo. Desses locais, era bombeado para a entrada do tratamento preliminar, composto por uma grade mecanizada (step- 
scream), caixa de areia e calha Parshall, em seguida o esgoto foi bombeado para o sistema piloto por meio de uma bomba submersa do modelo: NS 803, da marca Nine Star.

\subsection{Construção do sistema piloto}

A unidade experimental foi construída em tijolo maciço comum, com dimensões de $6 \mathrm{~m}$ de comprimento, 1,2 $\mathrm{m}$ de largura e 0,6 $\mathrm{m}$ de altura, dividida em 2 partes de $3 \mathrm{~m}$ de comprimento cada. A unidade foi construída ao nível do solo. A declividade do fundo foi de aproximadamente $1 \%$. Para garantir a impermeabilização do sistema, foi aplicada em toda a unidade experimental um revestimento impermeabilizante de base acrílica e o material suporte utilizado foi pedra britada com diferentes diâmetros.

\subsection{Wetland Construído de Fluxo Vertical (WCFV)}

A alimentação do WCFV foi realizada por meio de tubos de PVC (25 mm), perfurados em toda a sua extensão. Os tubos foram mantidos em suspensão sob o sistema, não tendo contato com o material filtrante, a fim de evitar a criação de caminhos preferenciais do líquido em relação ao material de enchimento. O sistema de drenagem no WCFV foi montado com tubos de PVC 50mm, perfurados nas laterais em toda extensão, em formato "L" rente ao fundo, com a função de coletar o esgoto percolado e encaminhá-lo para o Wetland de Fluxo Horizontal (WCFH). Na extremidade final do dreno, foi instalado um registro para controle de vazão, onde foi possível variar o TDH no WCFV e manter um fluxo de alimentação contínuo para o WCFH. O sistema de WCFV foi preenchido com $20 \mathrm{~cm}$ de pedra britada $\mathrm{n}^{\circ} 3 ; 20 \mathrm{~cm}$ de pedra britada $\mathrm{n}^{\circ} 2$ e $20 \mathrm{~cm}$ de pedra britada $\mathrm{n}^{\mathrm{o}} 1$, resultando em uma altura útil do leito de $60 \mathrm{~cm}$. A Figura 1 mostra um esquema do sistema de alimentação e drenagem do WCFV.

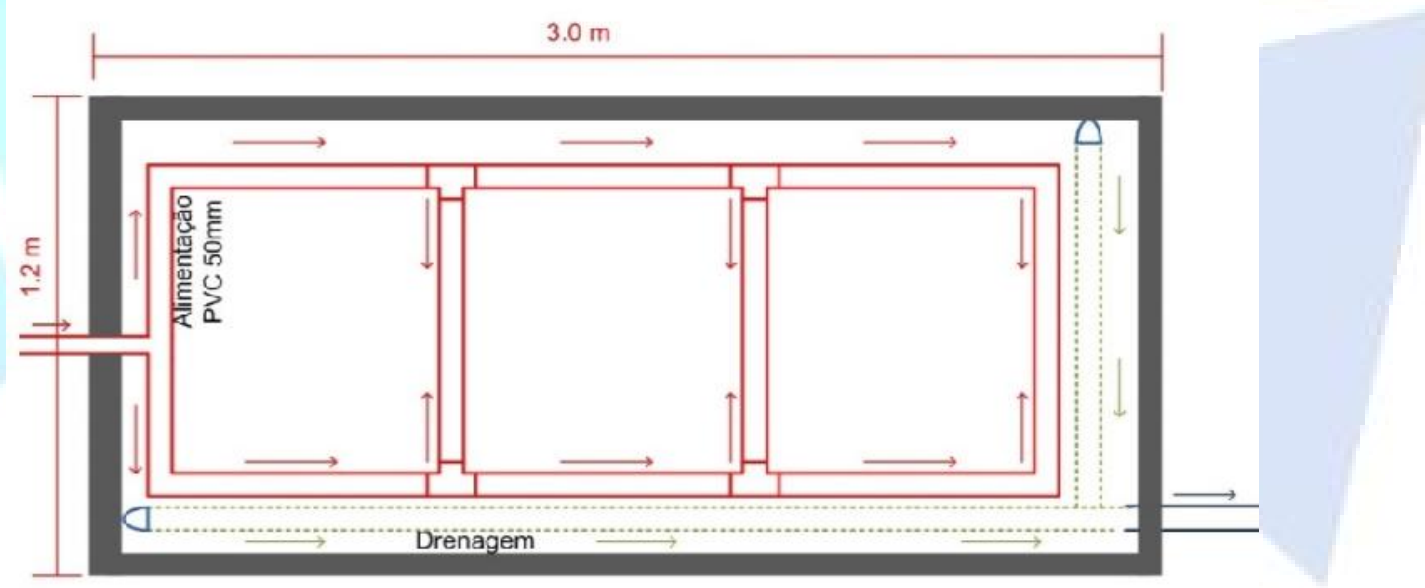

Figura 1. Esquema de alimentação e drenagem do Wetland Construído de Fluxo Vertical WCFV.

\subsection{Wetland Construído de Fluxo Horizontal (WCFH).}

O esgoto que alimentou o WCFH foi proveniente da WCFV. Este foi encaminhado por meio de uma bomba submersa, similar à da alimentação do WCFV. Essa alimentação foi realizada de forma contínua, fazendo com que o WCFH permanecesse submerso. No WCFH, próximo à saída do sistema, foi instalado um dreno em tubo PVC $40 \mathrm{~mm}$, perfurado, responsável por drenar o esgoto percolado horizontalmente e encaminhá-lo para fora do sistema. O WCFH teve como material de enchimento pedra britada $\mathrm{n}^{\mathrm{o}} 2$, por completo, com exceção às proximidades do dreno, que foram preenchidas com pedra de mão. A Figura 2 mostra o sistema de alimentação e drenagem da WCFH. 


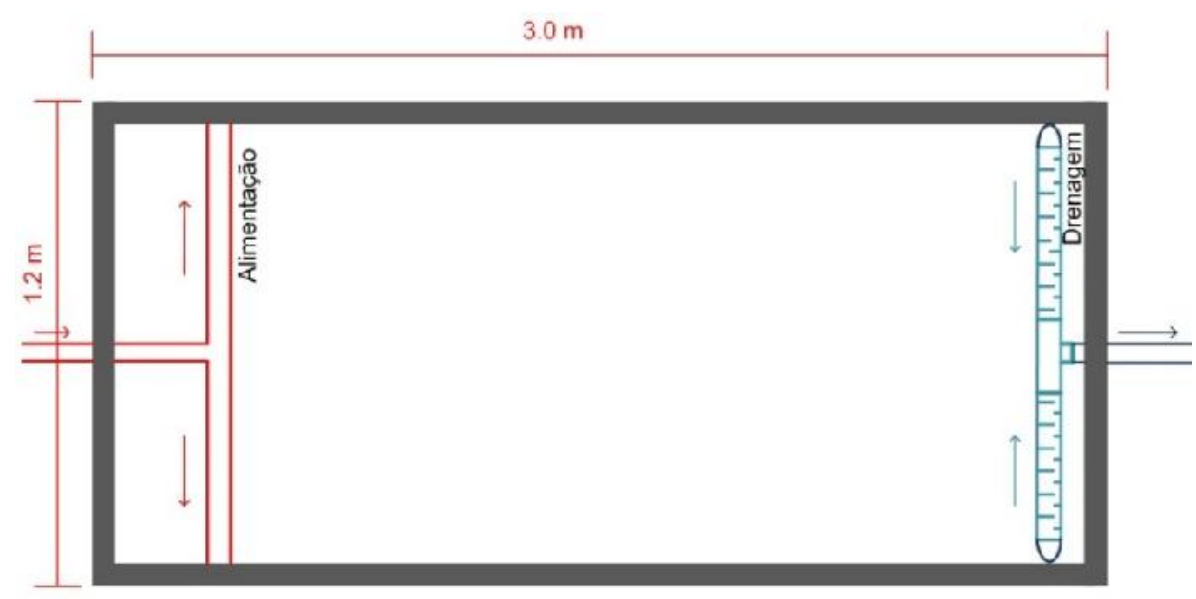

Figura 2. Esquema de alimentação e drenagem do Wetland Construído de Fluxo Horizontal WCFH.

\subsection{Macrófitas: Seleção e Plantio}

As macrófitas selecionadas foram do tipo emergente, que apresentam raízes presas ao fundo que permanecem parcialmente submersas e caule e folhas aéreos. Foram obtidas por meio de doação pelo viveiro Itubanaiá ${ }^{\circledR}$, localizado na cidade de Miracatu, estado de São Paulo. Para este estudo foram escolhidas cinco espécies de macrófitas emergentes de acordo com as recomendações de Lorenzi e Souza (2008). As mudas foram plantadas com raízes nuas, diretamente no material de enchimento obedecendo uma relação de \pm 4 mudas por $\mathrm{m}^{2}$ (CRITES, R. e TCHOBANOGLOUS, G., 1998).

\subsection{Condições de operação do sistema piloto}

A pesquisa foi desenvolvida em duas etapas. Na primeira foi considerada a fase de aclimatação do sistema. No "start up" o Wetland Construído Híbrido (WCH) foi alimentado com esgoto sanitário e feitas trocas volumétricas duas vezes por semana, permanecendo saturado para aperfeiçoar o desenvolvimento do biofilme no meio suporte e promover boa adaptação das macrófitas. Foi considerado o término na fase de aclimatação quando a eficiência da remoção de demanda química do oxigênio (DQO) atingiu valores superiores a 80\%, e as macrófitas encontravam-se adaptadas. $\mathrm{Na}$ segunda etapa, foi realizada a investigação experimental visando à remoção de material orgânico, nutrientes e organismos patogênicos. Conforme revisão da literatura a taxa de aplicação superficial (TAS) adotada foi entre 160 e $200 \mathrm{kgDBO} / \mathrm{ha}$.d (LETTINGA et al., 1993; SOUSA et al., 1996; MOLLE et al. 2006; HUA et al., 2010). A Tabela 1 mostra as principais características e condições de operação da WCH, durante a investigação experimental e a Figura 3 os pontos de amostragem no sistema.

Tabela 1 - Características operacionais do WCH.

\begin{tabular}{c|c}
\hline Variável & WCH \\
\hline Volume & $3960 \mathrm{~L}$ \\
\hline Volume Útil & $1777,1 \mathrm{~L}$ \\
\hline Área & $7,2 \mathrm{~m}^{2}$ \\
\hline Vazão & $200 \mathrm{~L} /$ dia \\
\hline Taxa de aplicação superficial de projeto & $200 \mathrm{KgDBO} / \mathrm{ha}$.dia \\
\hline Taxa de aplicação superficial efetiva & $214 \mathrm{KgDBO} / \mathrm{ha}$.dia \\
\hline Aplicações por dia & 8 \\
\hline Duração de cada aplicação & $3 \mathrm{~min}$ \\
\hline Intervalo entre as aplicações & $25 \mathrm{~L}$ \\
\hline Volume por aplicação & \\
\hline
\end{tabular}




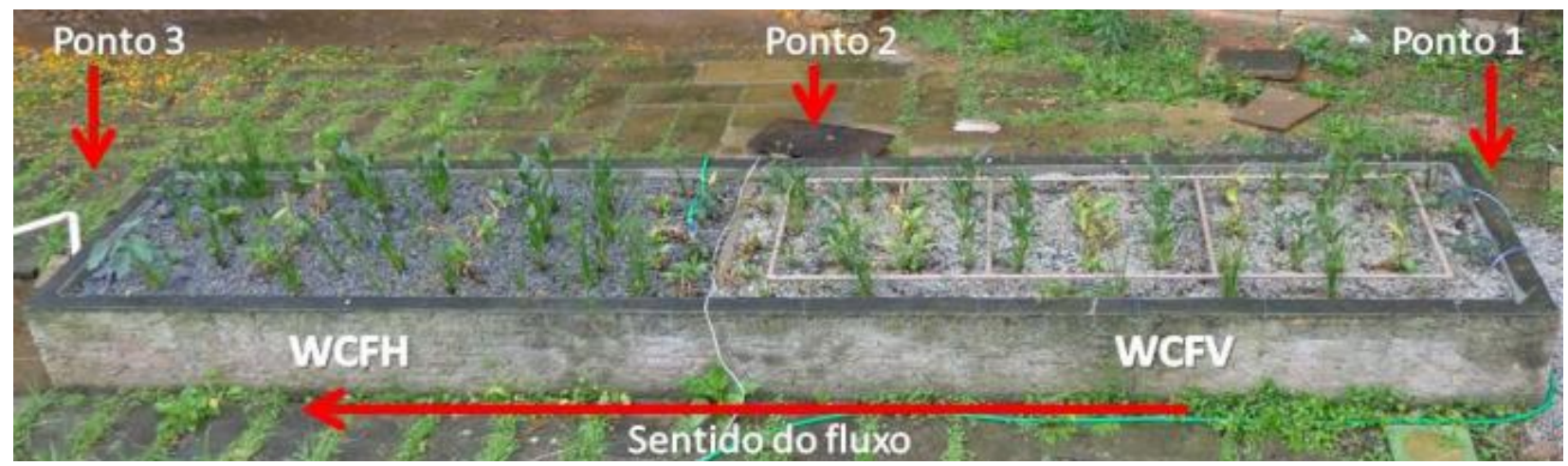

Figura 3. Pontos de amostragem realizadas no sistema de Wetland Construído. Legenda: Ponto 1: Afluente à WCH (esgoto pós tratamento preliminar); Ponto 2: Efluente do WCFV e Ponto 3: Efluente do WCFH.

\subsection{Ensaios laboratoriais}

As metodologias analíticas empregadas obedeceram a $22^{a}$ Edição do Standard Methods for Examination of Water and Wastewater, (APHA et al., 2012). As concentrações de nitrito e nitrato foram quantificadas em cromatógrafo de íons (Dionex-100, coluna ASCR2_mm e CSCR2_mm).

\section{RESULTADOS E DISCUSSÃO}

\subsection{Desenvolvimento das macrófitas e comportamento do WCH}

A fase de aclimatação do sistema de WCH durou um período de 14 semanas. Esse período compreendeu os meses de julho a outubro. As condições climatológicas desse período foram obtidas nos boletins mensais, disponibilizados pelo Instituto de Astronomia, Geofísica e Ciências Atmosféricas da Universidade de São Paulo (IAG, 2013). A Figura 4 mostra o desenvolvimento das macrófitas ao longo do período de estudo.

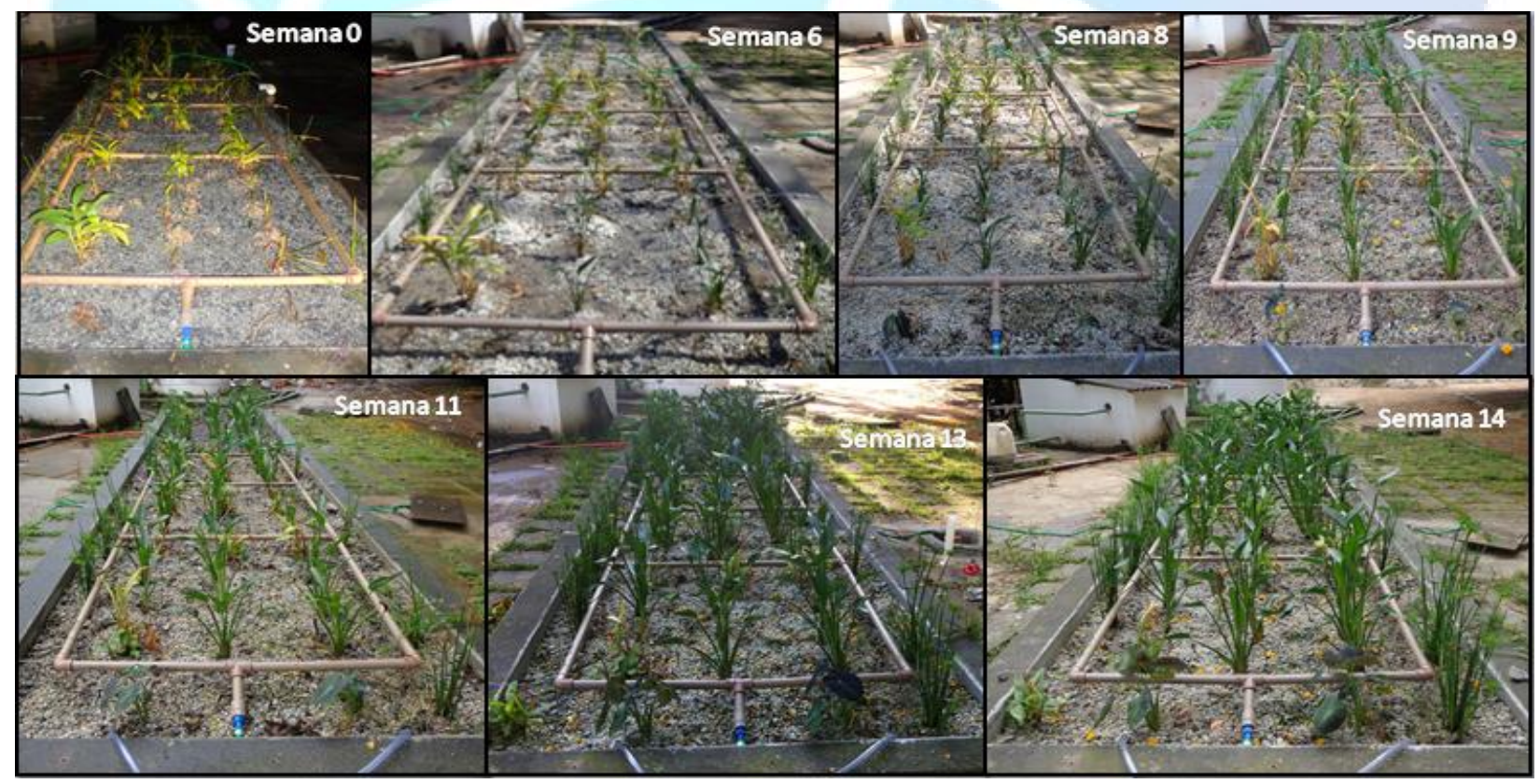

Figura 4 - Desenvolvimento das macrófitas ao longo do período de estudo. 


\subsection{Remoção de matéria orgânica}

Em relação à remoção de matéria orgânica, é possível observar na Tabela 2 que o sistema de WCH resultou um efluente com baixas concentrações de DQO com valores médios de 93,0 mg/L. Considerando que para esgoto sanitário a DBO é cerca da metade do valor da DQO, resultaria um valor de $\mathrm{DBO}<50 \mathrm{mg} / \mathrm{L}$ atendendo aos padrões de lançamento estabelecidos na Resolução nº430/2011 do CONAMA (CONAMA, 2011).

Tabela 2 - Valores médios de DQO Total nos efluentes do WCFV e WCFH.

\begin{tabular}{c|c|c|c}
\hline Variável & Esgoto Bruto & $\begin{array}{c}\text { Efluente } \\
\text { WCFV }\end{array}$ & $\begin{array}{c}\text { Efluente } \\
\text { WCFH }\end{array}$ \\
\hline Média (mg/L) & 664 & 288 & 93 \\
\hline Mínimo (mg/L) & 448 & 200 & 30 \\
\hline Máximo (mg/L) & 850 & 350 & 170 \\
\hline C.V. (\%) & 0,20 & 0,15 & 0,62 \\
\hline Desvio Padrão & 129,4 & 43,1 & 57,3 \\
\hline N & 9 & 9 & 9 \\
\hline
\end{tabular}

A Figura 5 mostra os dados da série histórica da eficiência de remoção de DQO obtida durante todo o estudo. Verifica-se que foi possível manter uma eficiência acima de $77 \%$ durante todo o estudo e uma eficiência média global de $87 \%$. Este valor encontra-se ligeiramente acima dos valores de eficiência de remoção encontrados nos estudos de Souza et al. (2000) com valores entre 79 e $86 \%$ e de Molle et al. (2006) que encontraram média de $82 \%$.

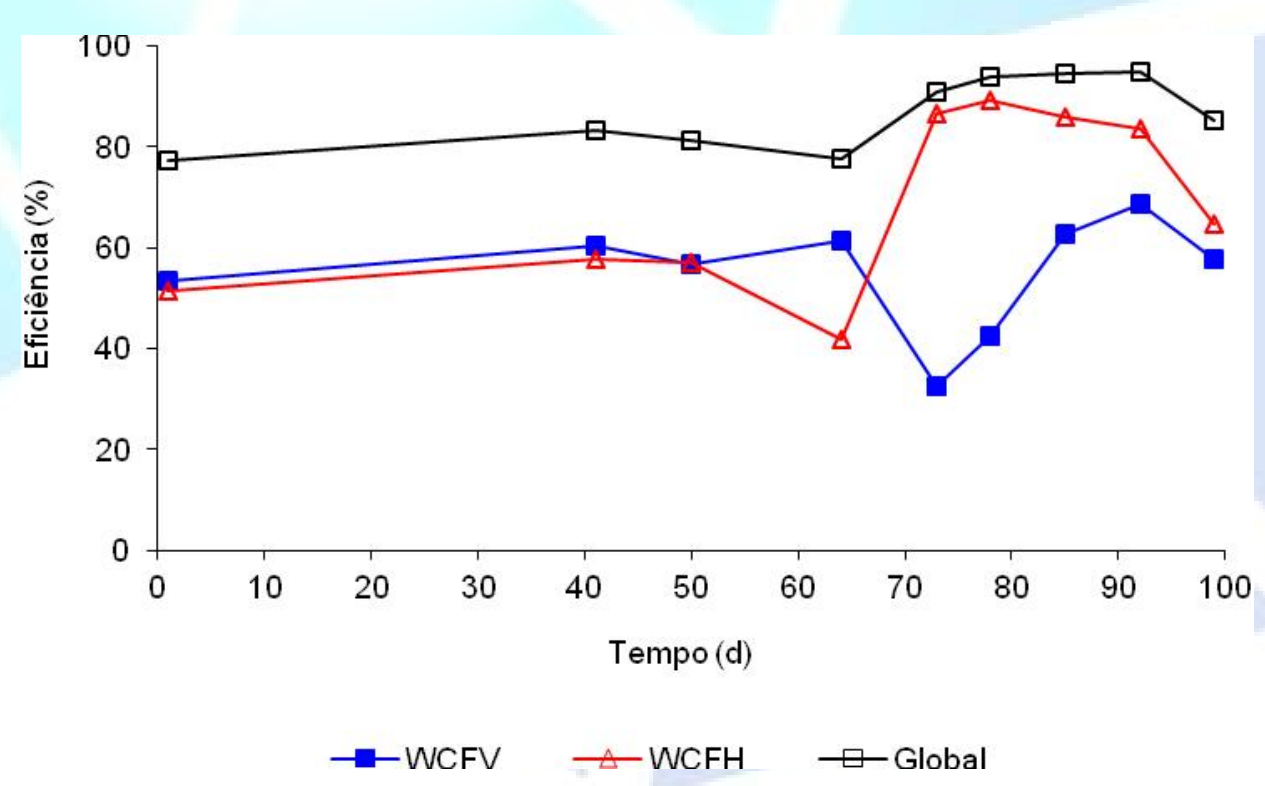

Figura 5. Série temporal da eficiência de remoção de DQO Total.

\subsection{Remoção de Nutrientes}

\subsubsection{Fósforo Total}

Em relação à remoção de fósforo, pode-se observar na Tabela 3 que o sistema de WCH resultou um efluente com baixas concentrações de fósforo total com valores médios de 1,8 mgP/L. Estes valores, no entanto, ainda podem ser limitantes para o lançamento em determinados corpos de água. 
Tabela 3 - Valores médios de fósforo total nos efluentes do WCFV e WCFH.

\begin{tabular}{c|c|c|c}
\hline Variável & Esgoto Bruto & $\begin{array}{c}\text { Efluente } \\
\text { WCFV }\end{array}$ & $\begin{array}{c}\text { Efluente } \\
\text { WCFH }\end{array}$ \\
\hline Média $(\mathrm{mgP} / \mathrm{L})$ & 5,5 & 3,3 & 1,8 \\
\hline Mínimo $(\mathrm{mgP} / \mathrm{L})$ & 4,1 & 2,2 & 1,1 \\
\hline Máximo $(\mathrm{mgP} / \mathrm{L})$ & 6,5 & 3,9 & 2,7 \\
\hline C.V.(\%) & 0,14 & 0,18 & 0,25 \\
\hline Desvio Padrão & 0,8 & 0,6 & 0,4 \\
\hline $\mathbf{n}$ & 9 & 9 & 9 \\
\hline
\end{tabular}

A Figura 6 mostra as eficiências de remoção de fósforo durante o período de estudo. A média deste fator obtida no WCFV foi de 38\%, valor abaixo do obtido nos estudos de Moraes (2012). Esse autor operou um WCFV de modelo francês tratando esgoto sanitário, resultando em eficiências de remoção de fósforo entre 45 e 50\%. A eficiência média global do WCH, porém, foi de $68 \%$, esse ganho em eficiência evidencia o benefício da associação dos fluxos.

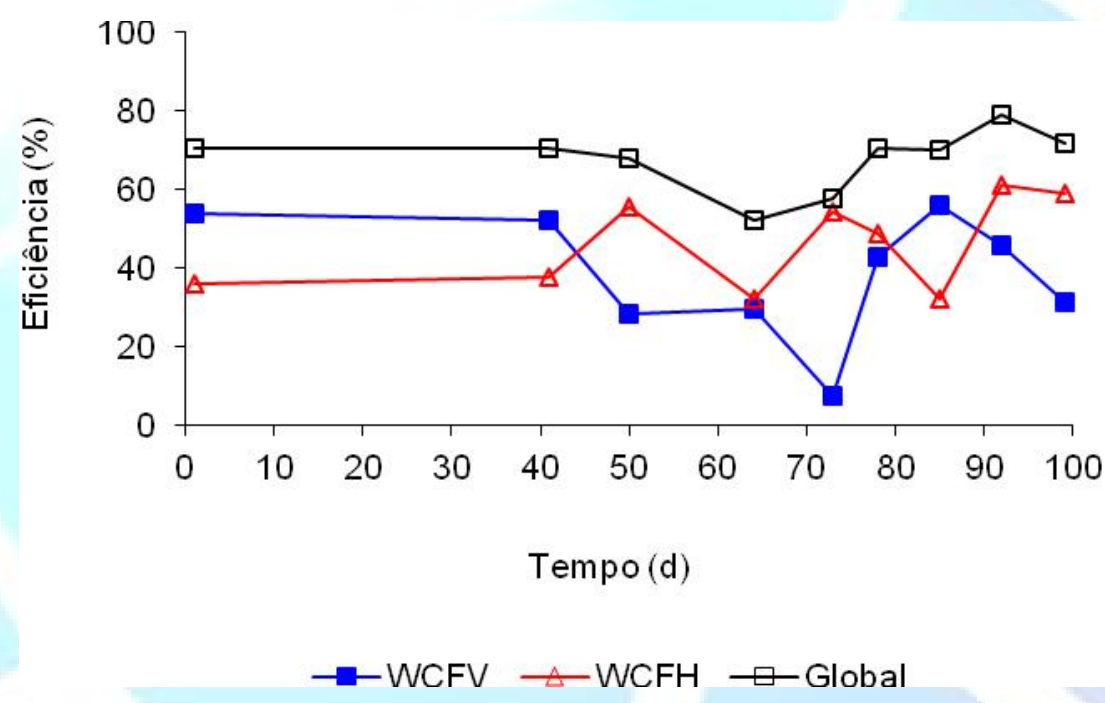

Figura 6. Valores das eficiências de remoção de fósforo total, durante o período de estudo.

\subsubsection{Remoção de material nitrogenado}

Em relação à remoção de nitrogênio, pode-se observar na Tabela 4 que o sistema resultou um efluente com concentrações médias de $\mathrm{NKT}_{\text {e }} \mathrm{NH}_{3}{ }^{-}$de 35,0 e 30,0 $\mathrm{mgN} / \mathrm{L}$, respectivamente. Estes valores ainda podem ser limitantes para o lançamento em determinados corpos de água, uma vez que de acordo com a Resolução CONAMA nº430/2011 (CONAMA, 2011) a concentração máxima de $\mathrm{NH}_{3}{ }^{-}$para lançamento em corpos hídricos é $20 \mathrm{mg} / \mathrm{L}$. Verificou-se que houve redução maior de NKT no WCFV, responsável por cerca de $20 \%$ dessa conversão. No estudo de Brix e Arias (2005), a nitrificação foi responsável por $49 \%$ da conversão da matéria nitrogenada. A Figura 7 mostra os resultados das concentrações de $\mathrm{NKT}^{-} \mathrm{NH}_{3}{ }^{-}$obtidas neste estudo. 
Tabela 4 - Valores médios de nitrogênio amoniacal nos efluentes do WCFV e WCFH.

\begin{tabular}{c|c|c|c|c|c|c}
\hline \multirow{2}{*}{ Variável } & \multicolumn{3}{|c|}{ NKT (mgN/L) } & \multicolumn{3}{c}{ NH $^{-}$(mgN/L) } \\
\cline { 2 - 7 } & $\begin{array}{c}\text { Esgoto } \\
\text { Bruto }\end{array}$ & $\begin{array}{c}\text { Efluente } \\
\text { WCFV }\end{array}$ & $\begin{array}{c}\text { Efluente } \\
\text { WCFH }\end{array}$ & $\begin{array}{c}\text { Esgoto } \\
\text { Bruto }\end{array}$ & $\begin{array}{c}\text { Efluente } \\
\text { WCFV }\end{array}$ & $\begin{array}{c}\text { Efluente } \\
\text { WCFH }\end{array}$ \\
\hline Média & 68 & 44 & 35 & 46 & 35 & 30 \\
\hline Mínimo & 53 & 22 & 21 & 32 & 24 & 17 \\
\hline Máximo & 81 & 62 & 44 & 56 & 44 & 40 \\
\hline C.V. (\%) & 0,15 & 0,32 & 0,27 & 0,15 & 0,22 & 0,25 \\
\hline Desvio Padrão & 10,0 & 14,2 & 9,5 & 6,8 & 7,6 & 7,3 \\
\hline n & 9 & 9 & 9 & 9 & 9 & 9 \\
\hline
\end{tabular}
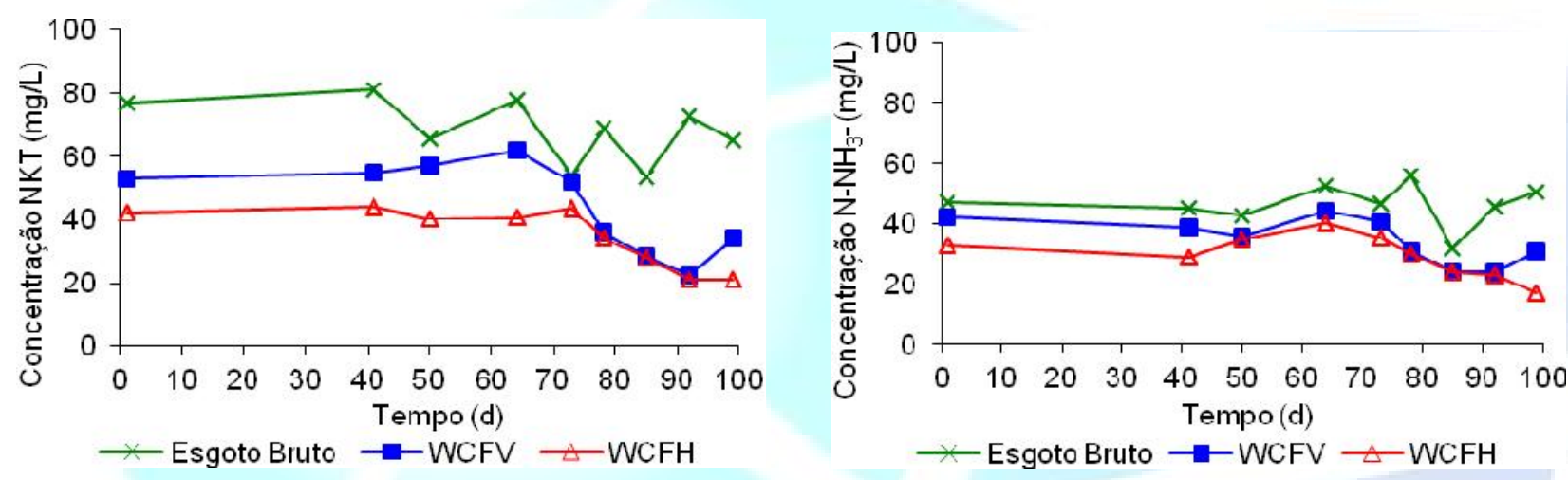

Figura 7. Valores das concentrações de NKT e N-amoniacal afluente e efluente ao WCFV e WCFH durante o período deste estudo.

$\mathrm{Na}$ Figura 8 se encontram os valores das eficiências obtidas de NKT e $\mathrm{NH}_{3}{ }^{-}$durante o período do estudo, sendo as médias globais de $48 \%$ e $35 \%$, respectivamente. Moraes (2012) obteve eficiência de $55 \%$ para NKT e $56 \%$ para $\mathrm{NH}_{3}{ }^{-}$, Molle et al. (2005) registraram eficiência média de $56 \%$ a $60 \%$ na remoção de NKT nos sistemas estudados, Olijnyk (2008) obteve remoção de 56\% a $74 \%$ de $\mathrm{NH}_{3}{ }^{-}$, e no estudo de Brix e Arias (2005) houve eficiência de $78 \%$ para $\mathrm{NH}_{3}{ }_{3}^{-}$. Esses valores mostram que os resultados de eficiência obtidos neste estudo estão abaixo do esperado. As concentrações de nitrato $\left(\mathrm{NO}_{3}^{-}\right)$e nitrito $\left(\mathrm{NO}_{2}{ }^{-}\right)$no esgoto bruto e no efluente da WCFV e WCFH apresentaram-se menores que 0,5 mgN/L. Esperavam-se maiores concentrações de $\mathrm{NO}_{3}{ }^{-}$no $\mathrm{WCFV}$. Devido ao baixo desenvolvimento do processo de nitrificação, no entanto, a oxidação da $\mathrm{NH}_{3}{ }^{-}$a $\mathrm{NO}_{3}{ }^{-}$não ocorreu com eficiência e, a parcela que foi oxidada não foi determinada no efluente da WCFH. Esta concentração de $\mathrm{NO}_{3}{ }^{-}$pode ter sido absorvida pelas macrófitas, que absorvem o nitrogênio em forma de íon amônio $\left(\mathrm{NH}_{4}{ }^{+}\right)$e de nitrato $\left(\mathrm{NO}_{3}{ }^{-}\right)$, sendo este último a via preferencial de absorção. 

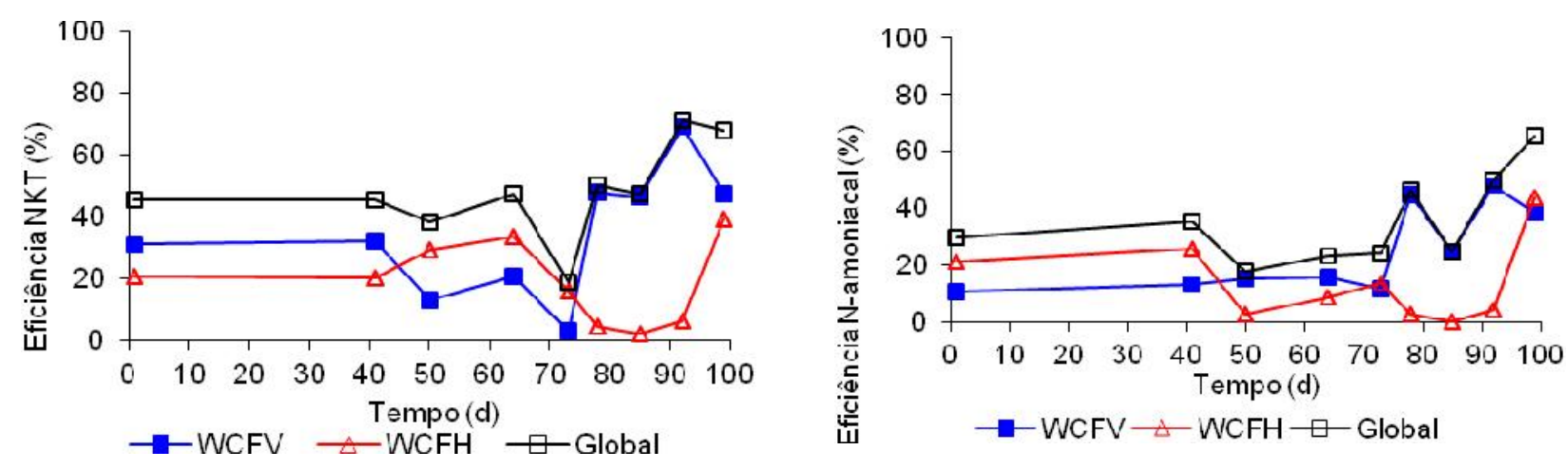

Figura 8. Série temporal da eficiência de remoção de NKT e N-amoniacal, durante o período de estudo.

\subsection{Turbidez}

Em relação à turbidez, foi possível observar que o WCH resultou efluente com baixa turbidez, de valores médios < 5,0 NTU (Tabela 5). A Figura 9 mostra as eficiências de turbidez obtidas durante este estudo. Observa-se que, independentemente das variações de turbidez do esgoto bruto, a turbidez no efluente final se manteve relativamente estável durante todo período do estudo.

Tabela 5 - Valores médios de Turbidez nos efluentes do WCFV e WCFH.

\begin{tabular}{c|c|c|c}
\hline Variável & Esgoto Bruto & $\begin{array}{c}\text { Efluente } \\
\text { WCFV }\end{array}$ & $\begin{array}{c}\text { Efluente } \\
\text { WCFH }\end{array}$ \\
\hline Média (NTU) & 122 & 19 & 5 \\
\hline Mínimo (NTU) & 82 & 9 & 2 \\
\hline Máximo (NTU) & 132 & 36 & 7 \\
\hline C.V. (\%) & 0,14 & 0,56 & 0,22 \\
\hline Desvio Padrão & 16,5 & 10,7 & 1,1 \\
\hline $\mathbf{n}$ & 8 & 8 & 8 \\
\hline
\end{tabular}
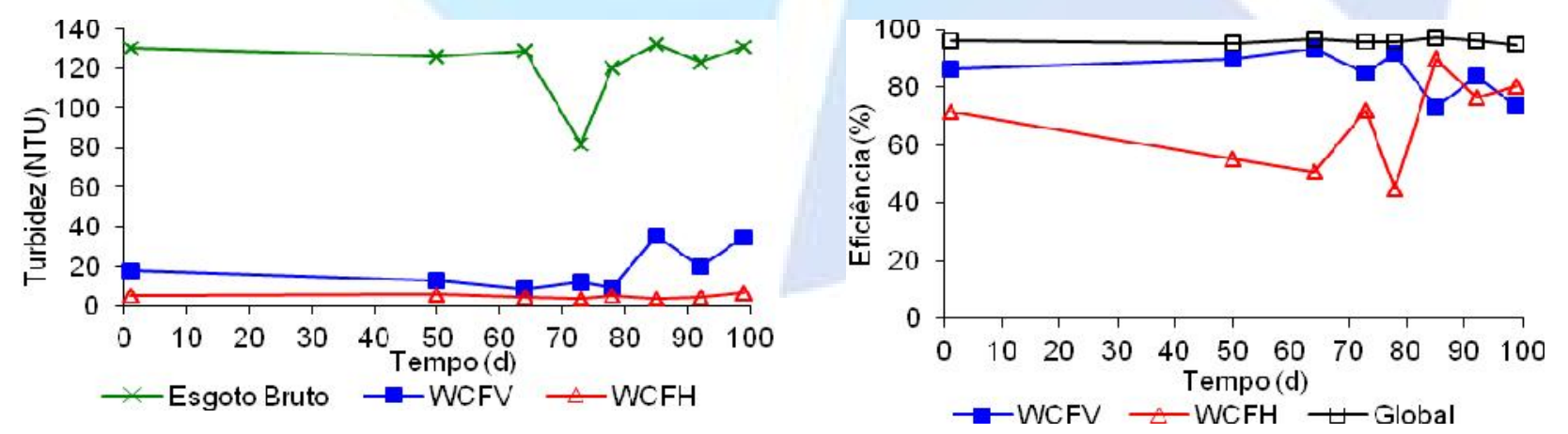

Figura 9. Valores de eficiência de turbidez no sistema estudado.

\subsection{Remoção de sólidos em suspensão no WCH}

Em relação à remoção de sólidos em suspensão totais (SST), é possível observar na Tabela 6 que o sistema de WCH resultou um efluente com baixas concentrações com valores médios de $12 \mathrm{e}$ 
$9 \mathrm{mg} / \mathrm{L}$. Este valor foi considerado baixo e mostra que o modelo proposto, utilizando somente britas como material de enchimento, não afetou a capacidade de retenção de sólidos no sistema.

Tabela 6 - Valores médios de SST, SSF e SSV nos efluentes do WCFV e WCFH.

\begin{tabular}{|c|c|c|c|c|c|c|c|c|c|}
\hline & \multicolumn{3}{|c|}{$\begin{array}{c}\text { Sólidos em Suspenção } \\
\text { Totais }\end{array}$} & \multicolumn{3}{|c|}{$\begin{array}{c}\text { Sólidos em Suspenção } \\
\text { Fixos } \\
\end{array}$} & \multicolumn{3}{|c|}{$\begin{array}{c}\begin{array}{c}\text { Sólidos em Suspenção } \\
\text { Voláteis }\end{array} \\
\end{array}$} \\
\hline & $\begin{array}{c}\text { Esgoto } \\
\text { Bruto }\end{array}$ & $\begin{array}{c}\text { Ef. } \\
\text { WCFV }\end{array}$ & $\begin{array}{c}\text { Ef. } \\
\text { WCFH }\end{array}$ & $\begin{array}{c}\text { Esgoto } \\
\text { Bruto }\end{array}$ & $\begin{array}{c}\text { Ef. } \\
\text { WCFV }\end{array}$ & $\begin{array}{c}\text { Ef. } \\
\text { WCFH }\end{array}$ & $\begin{array}{c}\text { Esgoto } \\
\text { Bruto }\end{array}$ & $\begin{array}{c}\text { Ef. } \\
\text { WCFV }\end{array}$ & $\begin{array}{c}\text { Ef. } \\
\text { WCFH }\end{array}$ \\
\hline Média & 2980 & 40 & 12 & 48 & 5 & 3 & 2932 & 35 & 9 \\
\hline Mínimo & 152 & 0 & 4 & 2 & 0 & 1 & 6 & 0 & 2 \\
\hline Máximo & 19420 & 66 & 26 & 242 & 8 & 6 & 19412 & 58 & 21 \\
\hline C.V. (\%) & 2,43 & 0,56 & 0,64 & 1,80 & 0,55 & 0,52 & 2,48 & 0,57 & 0,88 \\
\hline $\begin{array}{l}\text { Desvio } \\
\text { Padrão }\end{array}$ & 7249,7 & 22,6 & 7,7 & 86,0 & 2,5 & 1,7 & 7267,4 & 19,7 & 8,1 \\
\hline $\mathbf{N}$ & 7 & 7 & 7 & 7 & 7 & 7 & 7 & 7 & 7 \\
\hline
\end{tabular}

\section{CONCLUSÕES}

O Sistema de Wetlands Construído Híbrido se mostrou eficiente em relação à remoção de material orgânico, tendo eficiência média superior a 87\% para DQO e muito eficiente na remoção de sólidos, tendo um efluente clarificado, evidenciando assim a alta capacidade de retenção dos mesmos nos sistemas wetlands. Em relação a remoção de nutrientes, o sistema se mostrou satisfatório com redução de $48 \%$ de NTK e de $68 \%$ de fósforo total, estes valores foram próximos aos encontrados na literatura.

\section{REFERÊNCIAS}

APHA, AWWA, WEF. Standard Methods for examination of water and wastewater. Washington: American Public Health Association. EUA, 22 ${ }^{\text {nd }}$, ISBN 978-087553-013-0, 2012, $1360 \mathrm{p}$.

BRIX, H. Function of macrophytes in constructed wetlands. Water Science and Technology. EUA, v.29, n.4, pp. 71-78, 1994.

BRIX, H.; ARIAS, C. Danish guidelines for small-scale constructed wetland systems for on site treatment of domestic sewage. Water Science and Technology. EUA, v.51, n.9, pp.1-9, 2005.

CONSELHO NACIONAL DO MEIO AMBIENTE - CONAMA. Resolução nº 430, de 13 de maio de 2011. Dispõe sobre as condições de lançamento de efluentes, complementa e altera a Resolução $\mathrm{n}^{\circ}$ 357, de 17 de março de 2005, do Conselho Nacional do Meio Ambiente - CONAMA. 9 p. Disponível em: < http://www.mma.gov.br/>. Acesso em: 20 de setembro de 2015.

COOPER, P. A review of the design and performance of vertical-flow and hybrid reed bed treatment systems. Water Science and Technology. EUA, v.40, n.3, pp. 1-9, 1999. 
COOPER, P.; GRIFFIN, P.; HUMPHRIES, S.; POUND, A. Design of a hybrid reed bed system to achieve complete nitrification and denitrification of domestic sewage. Water Science and Technology. EUA, v.40, n.3, pp. 283-289, 1999.

CRITES, R.; TCHOBANOGLOUS, G. Small and Decentralized Wastewater Management Systems. Singapore: McGraw Hill, New York, v.1, 1084 p., 1998.

DIERBERG, F.E.; DEBUSK, T.A.; JACKSON, S.D.; CHIMNEY; M.J.; PIETRO, K. Submerged aquatic vegetation-based treatment wetlands for removing phosphorus from agricultural runoff: response to hydraulic and nutrient loading. Water Research. EUA, v.36, pp.1409-1422, 2002.

HUA, G.F.; ZHU, W.; ZHAO, L.F.; HUANG, J.Y. Clogging pattern in vertical-flow constructed wetlands: Insight from a laboratory study. Journal of Hazardous Materials. China, v.180, pp. 668-674, 2010.

IAG - USP. Resumo Mensal da Estação Meteorológica. 15a Edição. São Paulo, Setembro de 2013. Disponível em: < http://www.estacao.iag.usp.br/Mensais/Setembro2013.pdf>. Acesso em: 10 setembro, 2013.

LETTINGA G.; DEMAN A.; VANDERLAST A.R.M.; WIEGANT, W.; VANKNIPPENBERG, K.; FRIJNS, J.; VAN BUURENJ, C.L. Anaerobic treatment of domestic sewage and wastewater. Water Science and Technology. EUA, v. 27, n. 9, pp. 67-73, 1993.

LORENZI, H.; SOUZA, H.M. de. Plantas ornamentais no Brasil: arbustivas, herbáceas e trepadeiras. Nova Odessa: Instituto Plantarum. Brasil, 4. ed., 2008, 1088 p.

MASSOUD, M.A.; TARHINI, A.; NASR, J.A. Decentralized approaches to wastewater treatment and management: applicability in developing countries. Journal of environmental management. EUA, v.90, n.1, pp.652-659, 2009.

MOLLE, P.; LIÉNARD, A.; GRASMICK, A.; IWEMA, A. Effect of reeds and feeding operations on hydraulic behavior of vertical flow constructed wetlands under hydraulic overloads. Water Research. EUA, v.40, n.3, pp.606-612, 2006.

MORAES, D.C. Influência da sazonalidade e de plantas na redução da concentração de poluentes em sistema de alagados construídos de escoamento vertical, com alimentação em pulso, empregado para tratar esgoto doméstico bruto. 2012. 127 p. Dissertação (Mestrado em Engenharia Hidráulica) - Universidade Federal de Minas Gerais - UFMG, 2012. Disponível em: < http://www.bibliotecadigital.ufmg.br/dspace/handle/1843/ENGD-93DHWZ/>. Acesso em: 2015$10-04$.

OLIJNYK, D.P. Avaliação da nitrificação e desnitrificação de esgoto doméstico empregando filtros plantados com macrófitas (wetlands) de fluxos vertical e horizontal - sistemas híbridos. 2008. 113 p. Dissertação (Mestrado em Engenharia Ambiental) - Programa de Pós-Graduação em Engenharia Ambiental da Universidade Federal de Santa Catarina, Florianópolis, 2008. Disponível em: < https://repositorio.ufsc.br/bitstream/handle/123456789/91980/252380.pdf?sequence=1/>. Acesso em: 2015-10-04. 
SOUSA, J.T.; FORESTI, E. Domestic sewage treatment in an up-flow anaerobic sludge blanketsequential batch system. Water Science and Technology. EUA, v.33, n.3, pp.73-84, 1996.

SOUZA, J.T.; HAANDEL, A.C.; CABRAL, R.P.B. Desempenho de sistemas wetlands no póstratamento de esgotos sanitários pré-tratados em reatores UASB. Proceedings: IX Simpósio LusoBrasileiro de Engenharia Sanitária e Ambiental ABES. Porto Seguro- BA/Brasil, 9 a 14 de abril de 2000. III-072, CD-ROM. 